\title{
State Estimation and the Equivalence of the Regulatory and Supervisory Predictive Control Law
}

\author{
Damien Uduehi, Member, IEEE, Andrzej Ordys, Senior Member, IEEE, ${ }^{1}$ \\ and Michael Grimble, Fellow, IEEE
}

\begin{abstract}
This paper discusses the effect of state estimation on the equivalence between the regulatory and supervisory predictive control strategies for linear time invariant systems. The analysis presented here shows that in the presence of model-system mismatch, the use of a state estimator rather than the actual state in the feedback loop does not affect the equivalence between the two strategies.
\end{abstract}

Keywords : Supervisory, Predictive, State estimation.

\section{INTRODUCTION}

Model based predictive control is commonly applied as a supervisory dynamic optimization method, providing setpoint information to the PID controllers at the regulatory level. There are many studies that deal with set-point optimization via supervisory control, for instance Sáez, Cipriano and Ordys (2002), Ellis, Li and Riggs (1998), De Prada and Valentin (1996) and Katebi and Johnson (1997).

In this paper we consider the difference between the predictive control law at the regulatory level where the objective is to minimize the GPC cost index for direct multivariable control (Figure 1) and the predictive control law at the supervisory level, with the objective to minimize the GPC cost for supervisory indirect multivariable control (Figure 2). Uduehi et al (2003), showed that, under the assumption of state feedback, both strategies were equivalent. That analysis showed that even in the presence of model/system mismatch the equivalence was still guaranteed as long as the process output was identical to the estimated output.

As noted by Taylor et al (2002) one of the limitations with minimal state space representations, is that the state-vector is not normally accessible for direct measurement. An observer (Kalman 1960) has to be used to estimate the state which nominally converges asymptotically (Caines 1972) to the true state value. The results obtained in Uduehi et al (2003) are in this paper extended and combined with results from Ordys (1999) to show that in the presence of model system mismatch the output estimate from the observer converges to the true values, preserving the equivalence between the regulatory and supervisory control laws.

The paper is organized as follows, in section 2 the multivariable system is defined and the GPC control law for regulatory control is presented. In section 3, the GPC supervisory control law and the equivalence between both control laws is shown. In section 4 , the effect of modelsystem mismatch and of state estimation on the equivalence of the two algorithms is discussed. Simulation results using a process evaporator system model are presented in section 5 . The conclusions are given in section 6 .

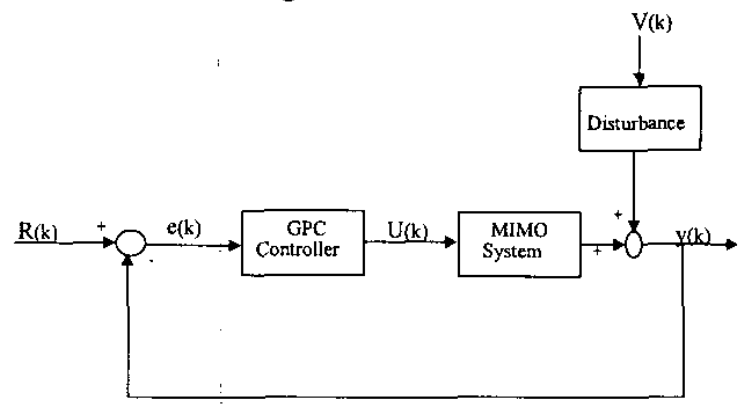

Figure 1 Regulatory Level GPC Control Structure

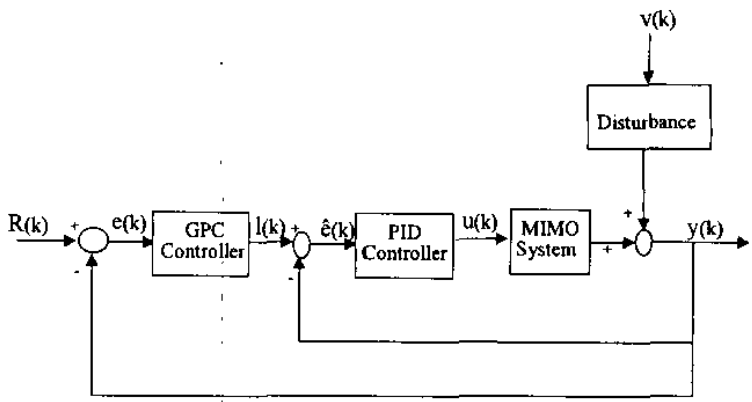

Figure 2 Supervisory Level GPC Control Structure

\section{GPC REGULATORY CONTROL LAW}

The derivation; of state-space multivariable GPC controller is now a routine and straightforward operation. It is outlined below, for completeness and to provide equations referenced in the following sections of the paper. For the direct multivariable GPC control structure shown in Figure 1, the plant is assumed to be linear, time invariant, minimum phase MIMO system containing no hidden unstable modes. The system is represented in state-space as

Manuscript received $14^{\text {th }}$ September 2003.

This work was partially supported by the Engineering and Physical Sciences Research Council under grant No. EPSRC GR/R04522/01

The authors are with the Industrial Control Centre, University of Strathclyde, 50 George Street, Glasgow G1 1QE, UK.

Corresponding author: A. Ordys tel.+44 1415484204, fax +44 1415484203,a.ordys@eee.strath.ac.uk 


$$
\begin{aligned}
& X_{k+1}=A_{1} X_{k}+B_{1} u_{k}^{5}+g_{1} v_{k+1} \\
& y_{k}=d_{1}^{T} X_{k}
\end{aligned}
$$

Where $u_{k}$ and $y_{k}$ are the system input and output vectors and $v_{k}$ is a vector of stochastic disturbances, acting on the system states. $v_{k}$ is assumed to be a Gaussian white noise source. The system is assumed to have $n$ states, $r$ inputs, $m$ outputs and $m$ disturbances. Notice that, in this paper, we assume noise-free measurement of the output.

Without loss of generality it is assumed that the number of states in the process is extended to accommodate all process time delays. It is easy to derive the output signal at time instant $k+j$, (where $k=1,2, \ldots \ldots \ldots N_{2}$ ). This output signal can be expressed as;

$$
y_{k+j}=d_{1}^{T} A_{1}^{j} X_{k}+\sum_{i=1}^{j} d_{1} A_{1}^{j-i} B_{1} u_{k+j-1}+\sum_{i=1}^{j} d_{1}^{T} A_{1}^{j-i} g_{1} v_{k+j}
$$

The GPC cost function $(J)$ is defined as the conditional expectation of the quadratic function of process errors and control actions, based on information available at time $k$ :

$$
\left.J=\mathrm{E}\left\{\sum_{j=1}^{N_{2}}\left(r_{k+j}-y_{k+j}\right)^{T} \phi_{e}\left(r_{k+j}-y_{k+j}\right)+\sum_{j=1}^{N_{k}} u_{k+j-1}{ }^{T} \phi_{u} u_{k+j-1}\right\}\right\} k
$$

Where $\phi_{\mathrm{e}}$ and $\phi_{\mathrm{u}}$ are weighting matrices.

From equation (3), if $\mathrm{N}_{1}=\mathrm{d}+1, \mathrm{~N}_{2}=\mathrm{N}$, the prediction horizon $\mathrm{Hp}=\mathrm{N}_{2}-\mathrm{N}_{1}+1$ and $\mathrm{Nu}=\mathrm{Hp}$, then the output prediction over this horizon can be written in vector notation as:

$\hat{\mathbf{Y}}_{R}=\mathbf{G} \hat{\mathbf{U}}_{R}+\mathbf{H} X_{k}$.

with:

$$
\mathbf{G}=\left[\begin{array}{cccc}
d^{T} B_{1} & 0 & \vdots & 0 \\
d_{1}^{T} A_{1} & d_{1}^{T} B_{1} & \vdots & \\
d_{1}^{T} A_{1}^{N-1} B_{1} & d_{1}^{T} A_{1}^{N-2} B_{1} & \vdots & d_{1}^{T} B_{1}
\end{array}\right], \quad \mathbf{H}=\left[\begin{array}{c}
d_{1}^{T} A_{1} \\
d_{1}^{T} A_{1}^{2} \\
\dddot{\ldots} \\
d_{1}^{T} A_{1}^{N}
\end{array}\right],
$$

Note that in solving the GPC cost function, because of the conditional expectation operator, all cross covariances associated with disturbances can be set to zero. The solution to this problem can be obtained by substituting the necessary parameters into the GPC criterion and solving for the required control coefficients as in Ordys and Clarke (1993).

$$
\hat{\mathbf{U}}_{R}=\left\{\mathbf{G}^{T} \Phi_{\mathrm{e}} \mathbf{G}+\Phi_{\mathrm{u}}\right\}^{-1}\left\{\mathbf{G}^{T} \Phi_{\mathrm{e}} \mathbf{R}-\mathbf{G}^{T} \Phi_{\mathrm{e}} \mathbf{H} X_{k}\right\}
$$

Because of the receding horizon strategy used in predictive control, notice that the control applied at the time instant $\mathrm{k}+1$ may of course differ from the prediction taken at time k, i.e $u_{k+1}\left|\hat{\mathbf{U}}_{k+1} \neq u_{k+1}\right| \hat{\mathbf{U}}_{k}$.

\section{GPC SUPERVISORY CONTROL LAW}

Consider the control of the process depicted in Figure 2. Let the MIMO system described by equations (1) and (2) be controlled by minimum phase, stable multi-loop PID controllers, as in Figure 2. Then it is necessary to make the assumption that the input and output pairings have been made and that the closed loop process with multi-loop PID controller in place contains no unstable hidden modes.

The discrete time state space representation for $\mathrm{i}^{\text {th }}$ PID controller can be written as:

$$
u_{k}^{i}=\left[\begin{array}{ll}
k_{i c} & k_{d c}
\end{array}\right] \tilde{x}_{k}^{i}+k_{k} e_{k}^{i}
$$

where, $k_{k}=k_{i c}+k_{d c}+k_{p c}$ and it is assumed that $k_{k}>0$,

$e_{k}^{i}=l_{k}^{i}-y_{k}^{i}$ and $\tilde{x}_{k+1}^{i}=A_{2}^{i} \tilde{x}_{k}^{i}+B_{2}^{i} e_{k}^{i}$

with the state equation dimensioned as follows; $x_{k}^{i}=2 \times 1, \quad A_{2}=2 \times 2, \quad B_{2}=2 \times 1$

Define:

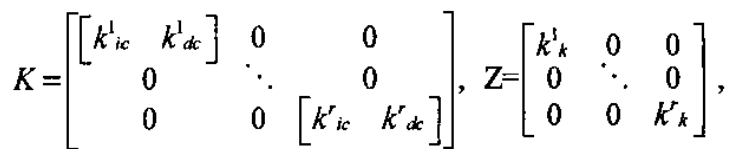

$$
\begin{aligned}
& A_{3}=\left[\begin{array}{ccc}
A_{2}^{1} & 0 & 0 \\
0 & \ddots & 0 \\
0 & 0 & A_{2}^{r}
\end{array}\right], B_{3}=\left[\begin{array}{ccc}
B_{2}^{1} & 0 & 0 \\
0 & \ddots & 0 \\
0 & 0 & B_{2}^{r}
\end{array}\right],\left[\begin{array}{c}
y_{k}^{1} \\
\vdots \\
y_{k}^{r}
\end{array}\right]=\Phi_{\mathrm{y}} d_{1}^{T} X_{k}
\end{aligned}
$$

Where $\Phi_{y}$ is a matrix designed to select the necessary outputs for feedback to the PID controllers. Then write the state equations for the multi-loop PID controller of the MIMO process as:

$$
\begin{aligned}
& u_{k}=K \tilde{x}_{k}+Z l_{k}-Z \Phi_{y} d_{1}^{T} X_{k} \\
& \tilde{x}_{k+1}=A_{3} \tilde{x}_{k}+B_{3} l_{k}-B_{3} \Phi_{y} d_{1}^{T} X_{k}
\end{aligned}
$$

\section{Remark:}

For controllers other than PID, for instance higher order controllers, it is still possible to write the equations in the state-space form of (11), (12), as long as the controller does not contain time delays. The only difference would be the dimensions of matrices in (8). Hence the proof that follows applies also to other types of controllers than PID.

Recall that the system state is defined by equation (1), then by extending the state of the system to include the state of the multi-loop PID controllers, a new state equation can be written. Defining:

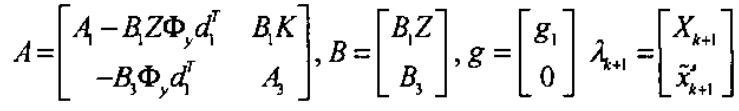

$$
\begin{aligned}
& P=\left[\begin{array}{ll}
-Z \Phi_{y} d_{1}^{T} & K
\end{array}\right], d=\left[\begin{array}{ll}
d_{1}^{T} & 0
\end{array}\right] \text { and } w=\Phi_{y} d_{1}^{T} \\
& \text { obtains: } \\
& \lambda_{k+1}=A \lambda_{k}+B l_{k}+g v_{k+1} \\
& y_{k}=d \lambda_{k} \\
& u_{k}=P \lambda_{k}+Z l_{k}
\end{aligned}
$$

The output signal at time instant $k+j$, (where $j=1,2, . . N_{2}$ ), can then be expressed as;

$y_{k+j}=d A^{j} \lambda_{k}+\sum_{i=1}^{j} d A^{j-i} B l_{k+i-1}+\sum_{i=1}^{j} d A^{j-i} g v_{k+i-1}$

While the input signal at time instant $k+j$, (where $\left.\mathrm{j}=0,2, \ldots \ldots \ldots N_{2}-1\right)$, can be expressed as; 
$u_{k+j}=P A^{j} \lambda_{k}+\sum_{i=1}^{j} P A^{j-i} B l_{k+i-1}+Z l_{k+j}+\sum_{i=1}^{j} P A^{j-i} g v_{k+i-1}$

Notice from equations (4), (15), (16) and (17) that $J$ is a quadratic function in both $u_{k}$ and $l_{k}$ and that $u_{k}$ and $l_{k}$ have a linear relationship. Hence it is possible to obtain the minimum by solving the GPC cost function with respect to future set-points $l_{k+j}, \mathrm{j} \varepsilon\left\{0 \mathrm{~N}_{2}-1\right\}$. For the process under supervisory GPC control, define the vector of future system outputs $\left(\mathbf{Y}_{S}\right)$, inputs $\left(\mathbf{U}_{S}\right)$ and set-points (L). From equations (18) and (19), with $\mathrm{N}_{1}=\mathrm{d}+1, \mathrm{~N}_{2}=\mathrm{N}$, the prediction horizon $\mathrm{Hp}=\mathrm{N}_{2}-\mathrm{N}_{1}+1$, and $\mathrm{N}_{\mathrm{u}}=\mathrm{Hp}$, the output and input signals over this horizon can be expressed as

$\mathbf{Y}_{s}=\mathbf{S L}+\mathbf{Q} \lambda_{k}$

$\mathbf{U}_{S}=\mathbf{M} \lambda_{k}+\mathbf{N L}$

The output and input predictions over this horizon can be expressed as

$\hat{\mathbf{Y}}_{s}=\mathbf{S} \mathbf{L}+\mathbf{Q} \lambda_{k}$ and $\hat{\mathbf{U}}_{s}=\mathbf{M} \lambda_{k}+\mathbf{N L}$

The matrices $\mathbf{S}, \mathbf{Q}, \mathbf{N}, \mathbf{M}$ and $\mathbf{D}$ are defined in the same way as $\mathbf{G}$ and $\mathbf{H}$ in the previous section.

The cost function can be written in vector notation as;

$J=\left\{\begin{array}{l}\left(\mathbf{R}-\mathbf{S L}-\mathbf{Q} \lambda_{k}\right)^{r} \Phi_{e}\left(\mathbf{R}-\mathbf{S L}-\mathbf{Q} \lambda_{k}\right) \\ +\left(\mathbf{M} \lambda_{k}+\mathbf{N L}\right) \Phi_{\nu}\left(\mathbf{M} \lambda_{k}+\mathbf{N L}\right)\end{array}\right\}$

Solving for the required control coefficients as in Ordys and Clarke (1993) obtains:

$\mathbf{L}=\left(\mathbf{S}^{T} \Phi_{e} \mathbf{S}+\mathbf{N}^{T} \Phi_{u} \mathbf{N}\right)^{-1}\left\{\mathbf{S}^{T} \Phi_{e} \mathbf{R}-\mathbf{S}^{T} \Phi_{e} \mathbf{Q} \lambda_{k}-\mathbf{N}^{T} \Phi_{u} \mathbf{M} \lambda_{k}\right\}$

To generalize the results to the case where model system mismatch exists, let the subscript $\square_{m o}$ denote the model parameters and the subscript $\square_{s y s}$ denote the system parameters, then the regulatory and supervisory control laws obtained in equations (6) and (24) can be more generally written as

$\hat{\mathbf{U}}_{R}=\left\{\mathbf{G}_{m o}^{T} \Phi_{\mathrm{e}} \mathbf{G}_{m o}+\Phi_{\mathrm{u}}\right\}^{-1} \quad\left\{\mathbf{G}_{m o}^{T} \Phi_{\mathrm{e}} \mathbf{R}-\mathbf{G}_{m o}^{T} \Phi_{\mathrm{e}} \mathbf{H}_{m o} X_{k}\right\}$

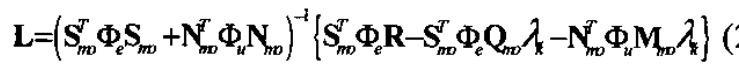

It was shown in Uduehi et al (2003), that:

$\mathbf{S}_{m o}=\mathbf{G}_{m o} \times \mathbf{N}_{m o}$

$\mathbf{Q}_{m o} \lambda_{k}=\mathbf{H}_{m o} X_{k}+\mathbf{G}_{m o} \mathbf{M}_{m o} \lambda_{k}$

Substituting equations (27) and (28) into equation (26), obtains as in Uduehi et al (2003):

$\mathbf{L}=\mathbf{N}_{m}^{-1}\left[\mathbf{G}_{m o}^{T} \Phi_{e} \mathbf{G}_{m o}+\Phi_{u}\right]^{-1} \mathbf{G}_{m v}^{T} \Phi_{e}\left(\mathbf{R}_{\zeta}-\mathbf{H}_{m} X_{k}\right)-\mathbf{N}_{m}^{-1} \mathbf{M}_{m} \lambda_{k}$

PID controllers do not require models, hence the multi-loop PID controller is model free and signal $\mathbf{U}_{s}$ is given by:

$\mathbf{U}_{s}=\mathbf{M}_{s y} \lambda_{k}+\mathbf{N}_{s y} \mathbf{L}+\mathbf{D}_{s y} \mathbf{V}$

Using equation (29) and (30), $\mathbf{U}_{S}$ can be written as:
$\mathbf{U}_{S}=\left\{\begin{array}{l}\mathbf{N}_{s y} \mathbf{N}_{m}^{-1}\left[\left(\mathbf{G}_{n v}^{T} \Phi_{e} \mathbf{G}_{n v}+\Phi_{u}\right)^{-1} \mathbf{G}_{m v}^{T} \Phi_{e}\left(\mathbf{R}-\mathbf{H} X_{k}\right)\right] \\ +\mathbf{M}_{s y} \lambda_{k}-\mathbf{N}_{s y} \mathbf{N}_{n v}^{-1} \mathbf{M}_{m v} \lambda_{k}+\mathbf{D}_{s y} \mathbf{V}\end{array}\right\}$

Then from equation (31), it can be observed that if,

$\mathbf{M}_{s y} \lambda_{k}=\mathbf{N}_{s y} \mathbf{N}_{m o}^{-1} \mathbf{M}_{m o} \lambda_{k}$ and $\mathbf{N}_{s y} \mathbf{N}_{m o}^{-1}=\mathbf{I}$

then the supervisory and regulatory level GPC controller would be equivalent. Notice that only the first row of (32) is used in calculating the current control action (receding horizon strategy). Analyzing the structure of matrices $\mathbf{M}$ and $\mathbf{N}$ and assuming that the parameters of the local controllers will be known, i.e. $Z_{m o}=Z_{s y}$, it is possible to deduce that first row of $\mathbf{N}_{s y} \mathbf{N}_{m o}^{-1} \mathbf{M}_{m o} \lambda_{k}$ will be $P_{m o} \lambda_{k}$. Then from equation (13),

$P_{m o} \lambda_{k}=\left[\begin{array}{ll}-Z \Phi_{y} d_{1 m o}^{T} X_{k} & K \tilde{x}_{k}\end{array}\right]$
$P_{s y} \lambda_{k}=\left[\begin{array}{ll}-Z \Phi_{y} d_{l s y}^{T} X_{k} & K \tilde{x}_{k}\end{array}\right]$

This implies that even if $A_{1 s y} \neq A_{1 m o}$ and $B_{1 s y} \neq B_{1 m o}$, then as long as $d_{1 s y}^{T} X_{k}=d_{1 m o}^{T} X_{k}$ and $K_{s y s} \tilde{x}_{k}=K_{m o} \tilde{x}_{k}$, then both the supervisory and regulatory GPC control strategies would be equivalent. Observe that $d_{1 s y}^{T} X_{k}=y_{k}$, and $d_{1 m o}^{T} X_{k}=\hat{y}_{k}$ (35)

From equation (35) the condition for equivalence can be formulated as a requirement that at any time instance $(\mathrm{k})$, the output of the process $\left(y_{k}\right)$ at that time instance, must be equivalent to the output obtained from the model.

\section{EFFECT OF STATE ESTIMATION ON CONTROL LAW EQUIVALENCE}

The analysis presented in section 3 showing the equivalence between the regulatory and supervisory control laws was made under the assumption of state feedback. Consider the case where access to the system state is unavailable. Then an observer is required, and in equations (25) and (29) the state can be replaced by the state estimate:

$$
\begin{aligned}
& \hat{\mathbf{U}}_{R}=\left\{\mathbf{G}_{m o}^{T} \Phi_{\mathrm{e}} \mathbf{G}_{m o}+\Phi_{u}\right\}^{-1}\left\{\mathbf{G}_{m o}^{T} \Phi_{\mathrm{e}} \mathbf{R}-\mathbf{G}_{m o}^{T} \Phi_{\mathrm{e}} \mathbf{H}_{m o} \hat{X}_{k}\right\} \\
& \mathbf{L}=\mathbf{N}_{m o}^{-1}\left[\mathbf{G}_{m o}^{T} \Phi_{e} \mathbf{G}_{m o}+\Phi_{u}\right]^{-1} \mathbf{G}_{m}^{T} \Phi_{e}\left(\mathbf{R}_{\mathrm{s}}-\mathbf{H}_{m} \hat{X}_{k}\right)-\mathbf{N}_{m o}^{-1} \mathbf{M}_{m} \hat{\lambda}_{k}
\end{aligned}
$$

The estimate of the system state can be calculated using standard Kalman, filter equations for a system without measurement noise:

$$
\begin{aligned}
& \hat{X}_{t+1 / t}=\left(A_{n v}-A_{m m} K_{t} D_{m o}\right) \hat{X}_{t / t-1}+A_{m o} K_{t} y_{t}+B_{m} u_{t} \\
& \hat{X}_{t+1 / t+1}=\hat{X}_{t+1 / t}+K_{t+1}\left[y_{t+1}-D_{m v} \hat{X}_{t+1 / t}\right] \\
& K_{t+1}=P_{t+1 / t} D_{m o}^{T}\left(D_{m o} P_{t+1 / t} D_{m o}^{T}\right)^{-1} \\
& P_{t+1 / t}=A_{m o} P_{t / t} A_{m o}^{T}+g_{m o} W g_{m o}^{T} \\
& P_{t+1 / t+1}=P_{t+1 / t}-P_{t+1 / t} D_{m o}^{T}\left(D_{m o} P_{t+1 / t} D_{m o}^{T}\right)^{-1} D_{m o} P_{t+1 / t}
\end{aligned}
$$


With the standard initial conditions.

Recall the condition of equivalence defined in equation (32). Under the assumption of output feedback this can be rewritten as:

$$
\mathbf{M}_{s y} \lambda_{k}=\mathbf{N}_{s y} \mathbf{N}_{m o}^{-1} \mathbf{M}_{m o} \hat{\lambda}_{k} \text { and } \mathbf{N}_{s y} \mathbf{N}_{m o}^{-1}=\mathbf{I}
$$

Using the same analysis as before the following observations can be made;

$$
\begin{aligned}
& P_{m o} \hat{\lambda}_{k}=\left[\begin{array}{ll}
-Z \Phi_{y} d_{1 m o}^{T} \hat{X}_{k} & K \hat{\tilde{x}}_{k}
\end{array}\right] \\
& P_{s y} \lambda_{k}=\left[\begin{array}{ll}
-Z \Phi_{y} d_{1 s y}^{T} X_{k} & K \tilde{x}_{k}
\end{array}\right]
\end{aligned}
$$

As before, the controller parameters as well as inputs to the regulatory level controller can be assumed known, and hence the controller state can be assumed to be known precisely such that even in the presence of disturbances $\tilde{x}_{k}=\hat{\tilde{x}}_{k}$. This leaves the condition $\hat{y}_{t}=y_{t}$.

Hence, to determine the effect of state estimation on the equivalence of the two control laws it is sufficient to analyse the output estimate from the Kalman filter. Using Caines (1972) and Ordys (1993), it can be shown that the Kalman filter converges to:

$P_{t / t}=0$

Substituting equation (46) into (41), obtain

$P_{t+1 / \ell}=g_{m o} W g_{m o}^{T}$

A check on the validity of equation (46), can be made by substituting (47), into (42), to see that

$$
\begin{aligned}
& P_{t+1 / t+1}=g_{m o} W g_{m o}^{T}-g_{m o} W g_{m o}^{T} D_{m o}^{T}\left(D_{m o} g_{m o} W g_{m o}^{T} D_{m o}^{T}\right)^{-1} D_{m o} g_{m o} W g_{m o}^{T} \\
& P_{t+1 / t+1}=g_{m o} W g_{m o}^{T}-g_{m o} W g_{m o}^{T} D_{m o}^{T}\left(g_{m o}^{T} D_{m o}^{T}\right)^{-1}(W)^{-1}\left(D_{m} g_{m o}\right)^{-1} D_{m o} g_{m o} W g_{m o}^{T} \\
& P_{t+1 / t+1}=g_{m o} W g_{m o}^{T}-g_{m} W g_{m o}^{T}=0
\end{aligned}
$$

Equation (48), shows that one possible solution to (42), is (46). The steady state Kalman gain $\left(K_{t+1}=K_{t}\right)$ can then be written using (47) as:

$$
\begin{aligned}
& K_{t+1}=g_{m o} W g_{m o}^{T} D_{m o}^{T}\left(D_{m o} g_{m o} W g_{m o}^{T} D_{m o}^{T}\right)^{-1} \\
& K_{t+1}=g_{m o} W g_{m o}^{T} D_{m o}^{T}\left(g_{m o}^{T} D_{m o}^{T}\right)^{-1}(W)^{-1}\left(D_{m o} g_{m o}\right)^{-1} \\
& K_{t+1}=g_{m o}\left(D_{m o} g_{m o}\right)^{-1}
\end{aligned}
$$

Recall that $\hat{y}_{t}=D_{m o} \hat{X}_{t / t}$, then using equation (49) and(39), deduce:

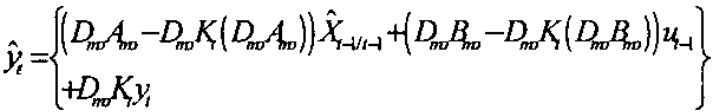

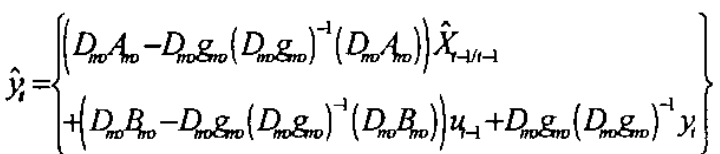

$$
\begin{aligned}
& \hat{y}_{t}=D_{m o} \hat{X}_{t / t}=y_{t}
\end{aligned}
$$

From equation (52), it can be observed that when a state estimator is used in predictive control design the conditions for equivalence between the regulatory and supervisory predictive control laws, even in the presence of mismatch are still met.

Remark: The assumption that the system has no measurement noise was necessary to derive the result. If this condition is not met the exact equivalence of the output and the output estimate cannot be guaranteed. However, it may still be possible that the mean values of the two signals are equal (Ordys, 1999). Then the difference between the two control strategies will be proportional to the power of the measurement noise.

\section{SUPERVISORY CONTROL OF AN EVAPORATOR WITH MODEL/SYSTEM MISMATCH}

In order to illustrate the equivalence between the regulatory and supervisory predictive control laws in the presence of model system mismatch and state estimation, Matlab simulations were carried out using the model of a forced circulation evaporator (Figure 3), as used the process industry (sugar mills, paper production, etc.).

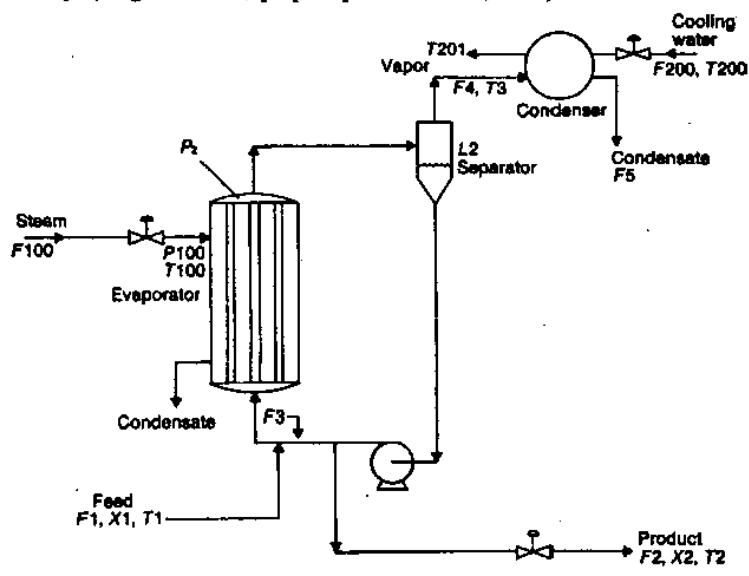

Figure 3 Evaporator System

Newell and Lee (1989) created a linearized model of the evaporator system with state matrices as shown below, and utilised it in a case study on controller design.

$\dot{X}(t)=A X(t)+B u(t)$ and $y(t)=C X(t)$

$A=\left[\begin{array}{ccc}0 & 0.10445 & 0.37934 \\ 0 & -0.1 & 0 \\ 0 & -0.01034 & -0.054738\end{array}\right], X(t)=\left[\begin{array}{c}L 2(t) \\ X 2(t) \\ P 2(t)\end{array}\right]$

$B=\left[\begin{array}{ccc}-0.1 & -.37266 & 0 \\ -0.1 & 0 & 0 \\ 0 & 0.036914 & -.0075272\end{array}\right], C=\left[\begin{array}{lll}1 & 0 & 0 \\ 0 & 1 & 0 \\ 0 & 0 & 1\end{array}\right]$

The process outputs to be controlled are defined as the separator level $\left(y_{1}(t)\right)$, the operating pressure $\left(y_{2}(t)\right)$ and the product composition $\left(y_{3}(t)\right)$. The control inputs into the process are defined as the product flow rate $\left(u_{1}(t)\right)$, the 
cooling water flow rate $\left(u_{2}(t)\right)$ and the steam pressure $\left(u_{3}(t)\right)$. For the simulation study, stochastic noise sources were added and the system equation was discretized so that: $X_{k+1}=A X_{k}+B u_{k}+g v_{k}$ and $y=d X_{k}$

The discrete transfer function used to generate all modelbased controllers was obtained using zero-order hold transformation and a sampling time $t_{s}=9$.

In order to illustrate the effect of state estimation with model/ system mismatch on the equivalence between the supervisory and regulatory predictive control laws the model of the process evaporator used for both the regulatory and supervisory predictive control design was mismatched from the actual system. For the system described by the state equation ;

$$
x_{k+1}=A_{s y} x_{k}+B_{s y} u_{k}+g_{s y} v_{k} \text { and } y=d_{s y} x_{k}
$$

The mismatch between the system and the design model was assumed to be adequately expressed by differences in the state matrices $B$, such that $B_{m o}=0.4 \times B_{s y}$. For the mismatched model, the output matrix $\left(d_{m o}\right)$ was chosen such that under state feedback $y_{k}=d_{m o} \hat{X}_{k}=d_{s y s} X_{k}$.

To verify the theoretical results obtained an experiment was set-up to compare the system responses and two different experiment scenarios were utilized to compare the simulated responses from the process under regulatory level GPC control and the process under supervisory level GPC. In both scenarios it was assumed that the state-vector was not normally accessible for direct measurement and a Kalman filter was implement for both controllers. For the first scenario the initial state of the system was assumed to be the same as the initial state of the Kalman filter, while in the second scenario the initial states of the system and Kalman filter are different.

The GPC design parameters used for both the supervisory and regulatory GPC controllers were chosen according to the guidelines in Newell and Lee (1989).

$\phi_{u 1}=0.1, \phi_{u 2}=0.5, \phi_{u 3}=0.2, \mathrm{~N}_{\mathrm{u}}=25, \quad \mathrm{~N}_{1}=1 \quad \mathrm{~N}_{2}=50$

For use with the supervisory GPC controller a nominal regulatory level controller was chosen to be a multi-loop PI controller using the tuning parameters from Newell and Lee (1989).

Figures 4 to 7 , show the process output and actuator responses to unit step change to the reference for the process as controlled by the nominal regulatory level controller (PID), regulatory level GPC controller and the supervisory GPC controller with a multi-loop PID inner loop. In Figure 4, the initial state of the state estimator and system were identical, while in Figure 5, there was a bias between the initial state of the state estimator and system. In Figures 6 and 7 a comparison is made between the two scenarios.

Comparing the results in Figures 4 to 8 , the following observations can be made:
- the signals in the system generated while using the regulatory GPC control strategy are exactly the same as produced by the supervisory GPC strategy, providing that the initial conditions for the state and for the Kalman filter are the same for both strategies. This does not necessarily mean that the initial conditions for the Kalman filter must be the same as for the state of the system

- while comparing the signals for the systems with different initial conditions on the state and on the Kalman filter we notice that, as expected, the outputs converge to the outputs obtained in the situation when the initial conditions for the state and for the Kalman filter are the same.
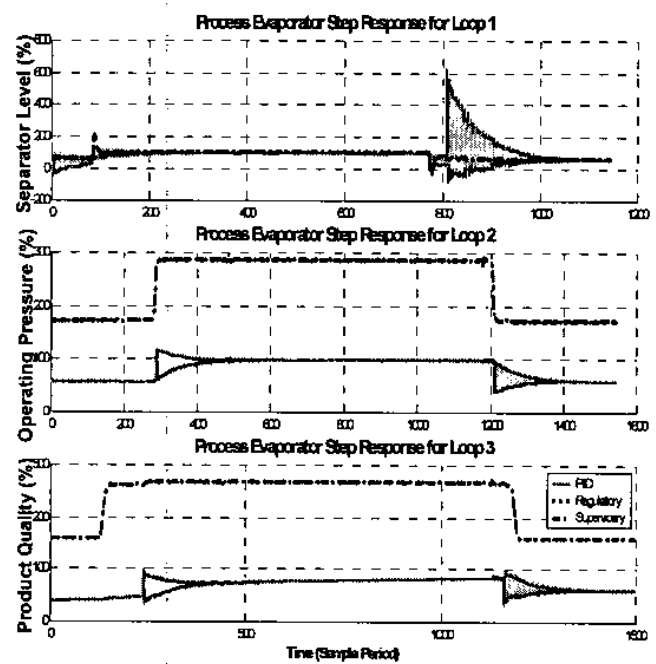

Figure 4 Evaporator Output Responses $\left(\hat{x}_{0}=x_{0}\right)$
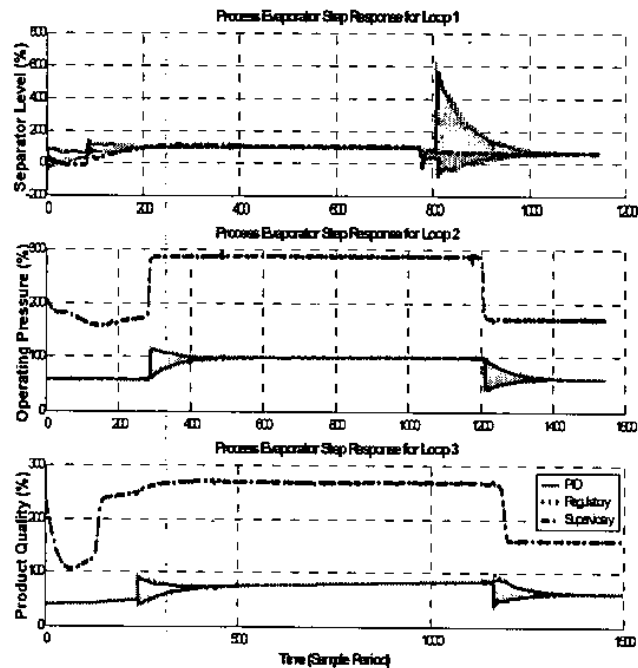

Figure 5 Evaporator Output Responses $\left(\hat{x}_{0} \neq x_{0}\right)$

These results verify the analytic results presented in section 3 , which showed that for the equivalence of regulatory and 
supervisory level predictive control law in the presence of mismatch, the condition was that equation (35) be valid. As shown in section 4 , using a state estimator guarantees that the estimated process output is equal to the actual output from the process (equation (52)) and hence satisfies equation (35).
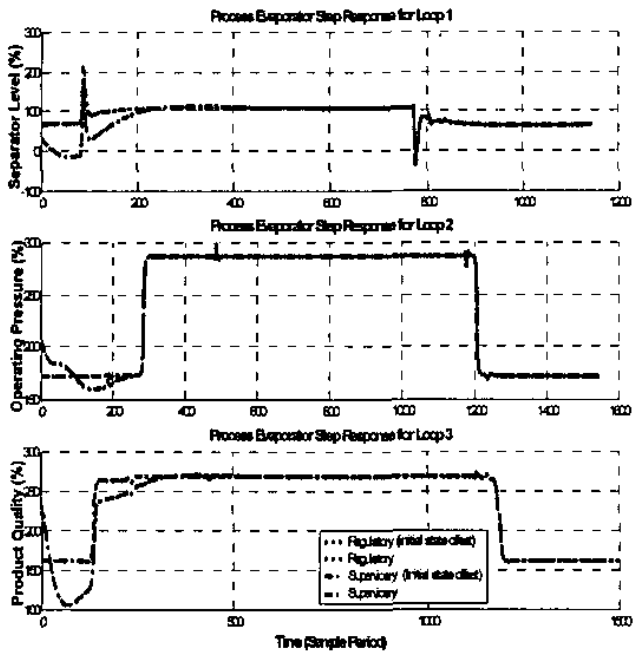

Figure 6 Evaporator Output Resp. $\left(\hat{x}_{0}=x_{0}\right.$ and $\left.\hat{x}_{0} \neq x_{0}\right)$
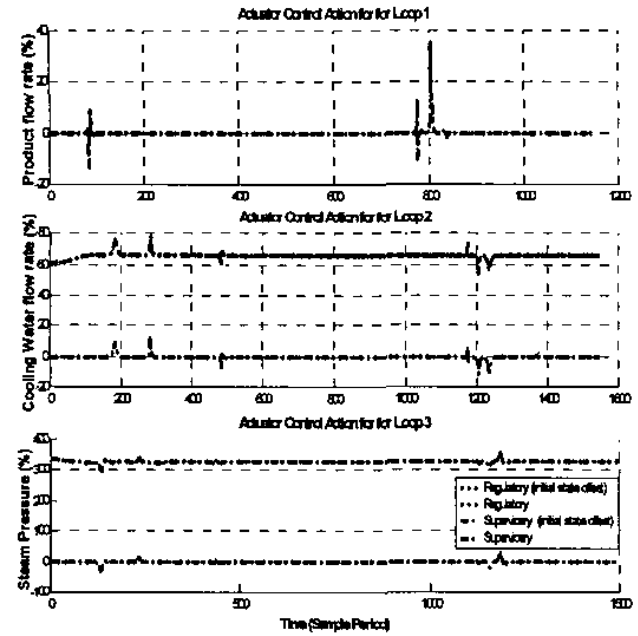

Figure 7 Evaporator Input Responses $\left(\hat{x}_{0}=x_{0}\right.$ and $\hat{x}_{0} \neq x_{0}$ )

However, one may notice from figure 9 that the inputs for the situation of different conditions for the state and for the Kalman filter do not converge to the inputs for the situation of the same initial condition for the system and for the Kalman filter. This property was not guaranteed by the theory presented in chapter 3 and indeed, for this particular system one may check that the same steady-state outputs may be produced by different combinations of steady-state inputs. (The reader is reminded that the equivalence between the supervisory and the low-level structure still holds.)

\section{CONCLUSIONS}

The analysis presented in this paper shows that an inner PID regulatory loop offers no additional advantage when using dynamic supervisory set-point optimization via GPC control. The supervisory GPC controller completely cancels out the PID controller(s) dynamics even for multivariable systems with model system mismatch and state estimation. As extension of the results of this paper, it can also be showed that the equivalence holds for systems with constraints.

We are not claiming that the PID level should be completely removed but that one should be aware of that equivalence and, perhaps, when tuning PID controllers put more attention to those loops which are not supervised.

\section{REFERENCES}

[1] Clarke D. W. and Gawthrop P. J., 1975a, Simulation of a generalised self-tuning regulator, Electronics Letters, 11(2), 41-2

[2] Clarke D. W. and Gawthrop P. J., 1975b, Self-tuning controller, IEE Proceedings Part D: Control Theory and Applications, 122(9) pp 929-934

[3] De Prada C. and Valentin A., 1996, Set point optimisation in multivariable constrained predictive control, Proceedings of the $13^{\text {th }}$ Congress of IFAC, San Francisco pp. 351-356

[4] Ellis R., Li X and Riggs J., 1998, Modelling and optimisation of a model IV fluidised catalytic cracking unit, AIChE Journal, vol 44, no. 9, pp 2068-2079

[5] Giovanini L, 2001, Embedded Estimator in Model Predictive Control, $2^{\text {nd }}$ Latin American Workshop on Testing and Fault Tolerance Systems, pp 230-239, Cancun Mexico

[6] Giovanini L, 2003, Predictive Feedback Control, ISA Trans. J, vol 42(2) pp 207-226

[7] Katebi M. and Johnson M, 1997, Predictive control design for large-scale systems. Automatica, vol 33, 421-425

[8] Kalman R, 1960, A new approach to linear filtering and Prediction problems. ASME Trans. J Basic Eng. 82D

[9] Ordys A.W., Steady-state offset in predictive control, American Control Conference, San Diego, USA, June 1999

[10] Ordys A., 1993, Model System Parameter Mismatch In GPC Control, Int. J.of Control, vol 7, pp 239-253

[11] Ordys A and Clarke D., 1993, A state space description of GPC controller; Int J. of Systems Sci, vol 24(9), 1727-44

[12] Saez D., Cipriano A, Ordys A., 2002, Optimisation of Industrial Processes at The Supervisory Level, Advances in Industrial Control, Springer-Verlag London

[13] Bitmead R., Gevers M. and Wertz V, 1990, Adaptive Optimal Control The Thinking man's GPC. Prentice Hall

[14] Taylor C. J, Chotai A. and Young P. C, 2000, State space control system design based on non-minimal state-variable feedback: further generalization and unification results. Int. J. of Control, vol 73, No. 14, pp 1329 - 1345

[15] Uduehi D., Ordys A., Grimble M, 2003, Supervisory and Regulatory Predictive Control Law Equivalence, Submitted to Automatica 2003.

[16] Newell R. B and Lee P. L, 1989, Applied Process Control; A Case Study. Prentice Hall 